\title{
27-Hydroxycholesterol induces hematopoietic stem cell mobilization and extramedullary hematopoiesis during pregnancy
}

\author{
Hideyuki Oguro, ${ }^{1,2,3}$ Jeffrey G. McDonald, ${ }^{4}$ Zhiyu Zhao, ${ }^{1,2}$ Michihisa Umetani, ${ }^{5}$ Philip W. Shaul, ${ }^{2,6}$ and Sean J. Morrison ${ }^{1,2,7}$ \\ 'Children's Research Institute and ${ }^{2}$ Department of Pediatrics, University of Texas Southwestern Medical Center, Dallas, Texas, USA. ${ }^{3}$ Cellular Engineering, The Jackson Laboratory for Cenomic Medicine, \\ Farmington, Connecticut, USA. ${ }^{4}$ Department of Molecular Cenetics, University of Texas Southwestern Medical Center, Dallas, Texas, USA. ${ }^{5}$ Center for Nuclear Receptors and Cell Signaling, \\ Department of Biology and Biochemistry, University of Houston, Houston, Texas, USA. ${ }^{6}$ Center for Pulmonary and Vascular Biology and ${ }^{7}$ Howard Hughes Medical Institute, University of Texas \\ Southwestern Medical Center, Dallas, Texas, USA.
}

\begin{abstract}
Extramedullary hematopoiesis (EMH) is induced during pregnancy to support rapid expansion of maternal blood volume. EMH activation requires hematopoietic stem cell (HSC) proliferation and mobilization, processes that depend upon estrogen receptor $\alpha(E R \alpha)$ in HSCs. Here we show that treating mice with estradiol to model estradiol increases during pregnancy induced HSC proliferation in the bone marrow but not HSC mobilization. Treatment with the alternative ERa ligand 27-hydroxycholesterol (27HC) induced ER $\alpha$-dependent HSC mobilization and EMH but not HSC division in the bone marrow. During pregnancy, 27HC levels increased in hematopoietic stem/progenitor cells as a result of CYP27A1, a cholesterol hydroxylase. Cyp27a1-deficient mice had significantly reduced 27HC levels, HSC mobilization, and EMH during pregnancy but normal bone marrow hematopoiesis and EMH in response to bleeding or G-CSF treatment. Distinct hematopoietic stresses thus induce EMH through different mechanisms. Two different ER $\alpha$ ligands, estradiol and 27HC, work together to promote EMH during pregnancy, revealing a collaboration of hormonal and metabolic mechanisms as well as a physiological function for $27 \mathrm{HC}$ in normal mice.
\end{abstract}

\section{Introduction}

Adult hematopoietic stem cells (HSCs) reside primarily in the bone marrow, and their number is tightly regulated under steadystate conditions. However, hematopoietic stresses promote HSC proliferation and mobilization to extramedullary tissues, including the spleen, to expand the number of HSCs and increase hematopoiesis (1). The mechanisms that regulate HSC function under steady-state conditions have been extensively characterized, but the mechanisms that regulate HSC activation in response to hematopoietic stresses, such as pregnancy, are poorly understood.

HSCs divide more often in female as compared with male mice (2). Estrogen receptor $\alpha(E R \alpha)$, but not ER $\beta$, is highly expressed by HSCs, and 17 $\beta$-estradiol (E2), an endogenous estrogen agonist of $\mathrm{ER} \alpha$, increases HSC division in female mice. E2 levels increase during pregnancy, when extramedullary hematopoiesis $(\mathrm{EMH})$ is activated to increase the production of red blood cells. A loss of EMH during pregnancy reduces maternal blood cell counts (3). Maternal EMH during pregnancy requires increased HSC proliferation in the bone marrow, mobilization to the spleen, and splenic erythropoiesis, processes that depend upon $\mathrm{ER} \alpha$ function in HSCs (2).

Sex hormones, such as E2, are not the only endogenous ligands for estrogen receptor. 27-Hydroxycholesterol $(27 \mathrm{HC})$, an oxyster$\mathrm{ol}$, also binds to estrogen receptors and regulates their function (4).

Conflict of interest: The authors have declared that no conflict of interest exists. Submitted: March 16, 2017; Accepted: June 15, 2017

Reference information: / Clin Invest. 2017;127(9):3392-3401.

https://doi.org/10.1172/JCI94027.
Oxysterols are metabolites of cholesterol, and $27 \mathrm{HC}$ is the most abundant oxysterol in mice (5). Plasma $27 \mathrm{HC}$ levels strongly correlate with total cholesterol levels (6), as $27 \mathrm{HC}$ is generated directly from cholesterol by the sterol hydroxylase CYP27A1. Plasma $27 \mathrm{HC}$ levels are greatly reduced in Cyp27a1 $/$ mice (7). CYP27A1 is abundant in the liver, where $27 \mathrm{HC}$ is produced as a substrate for bile acid synthesis, but it is also expressed in non-hepatic tissues (5). In the context of dietary or genetic changes that elevate $27 \mathrm{HC}$ levels, 27HC can modulate $\mathrm{ER} \alpha$ function to inhibit vascular repair in cardiovascular disease (4), promote ER-positive breast cancer growth $(8,9)$, and increase the severity of atherosclerosis (10). However, it has been unclear whether $27 \mathrm{HC}$ has a physiological signaling function in normal mice.

Although ER $\alpha$ regulates HSC function (2) and $27 \mathrm{HC}$ is an ERo ligand (4), it has not been tested whether 27HC regulates HSCs. Nonetheless, cholesterol is known to promote HSC proliferation and mobilization (11-13). Patients with hypercholesterolemia mobilize larger numbers of $\mathrm{CD} 34^{+}$cells following treatment with cyclophosphamide and granulocyte colony-stimulating factor (G-CSF) as compared with patients with lower cholesterol levels (14). Mice with defects in cholesterol efflux as a result of $A b c a 1$ and $A b c g 1$ transporter deficiency display increased hematopoietic stem and progenitor cell (HSPC) numbers, proliferation, and mobilization $(15,16)$. The cholesterol transporters influence HSPC function through cellautonomous and non-cell-autonomous mechanisms (15), though our understanding of these mechanisms remains limited.

In this study we show that the cholesterol metabolite $27 \mathrm{HC}$ acts directly on HSCs to promote their mobilization in an ER $\alpha$ - 
A

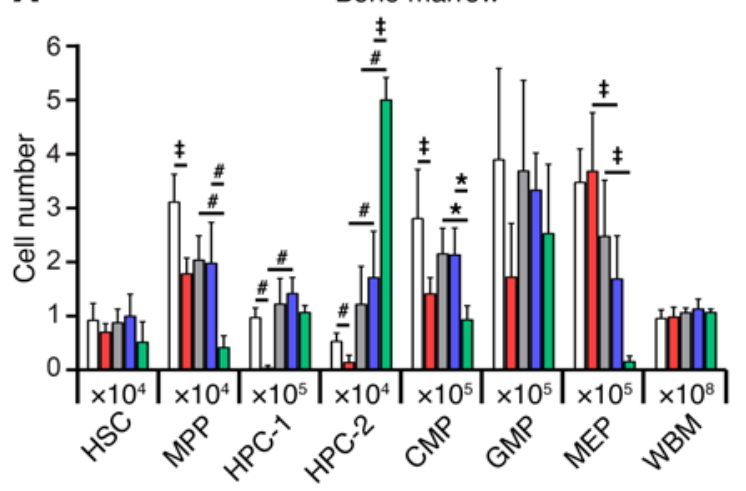

B

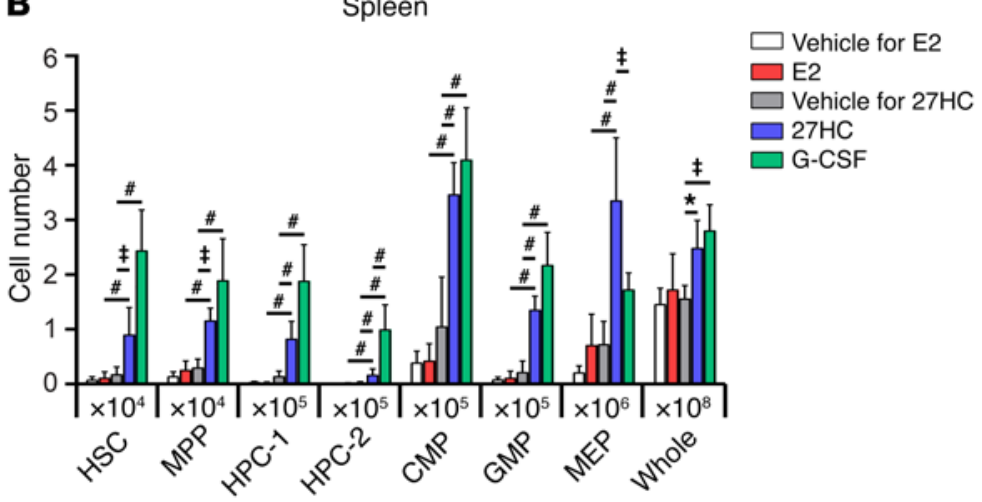

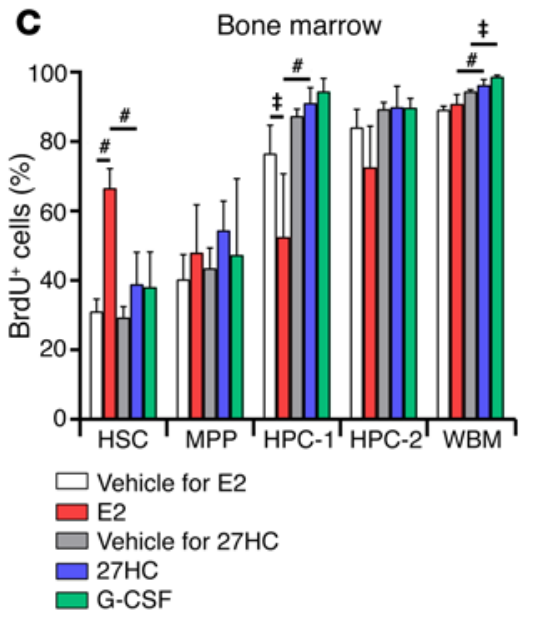

D

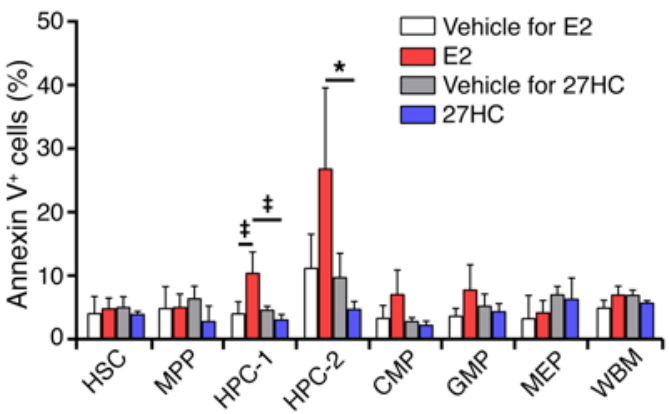

E

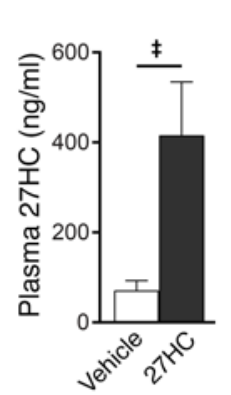

$\mathbf{F}$

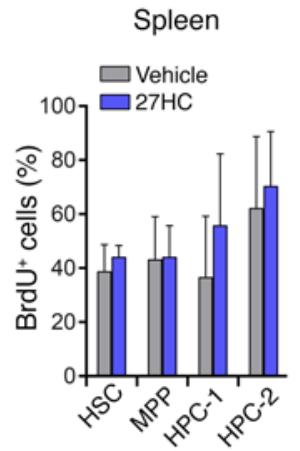

Figure 1. Estradiol promotes HSC division in the bone marrow and 27HC promotes mobilization to the spleen. (A and B) The numbers of hematopoietic stem and progenitor cells in the bone marrow (femurs and tibias; $\mathbf{A}$ ) and spleen (B) of male mice treated with estradiol (E2), 27HC, or G-CSF daily for 6 days (a total of 4-5 mice/treatment from 5 independent experiments). The vehicle for E2 was corn oil, and the vehicle for 27HC was 2-hydroxypropyl$\beta$-cyclodextrin. The markers used to identify each cell population are shown in Methods. (C) BrdU incorporation into hematopoietic stem and progenitor cells in the bone marrow of male mice treated with E2, 27HC, or G-CSF for 6 days. The mice received BrdU for the last 3 days (a total of 4-5 mice/treatment from 4 independent experiments). (D) The frequency of annexin $\mathrm{V}^{+}$cells in the indicated hematopoietic stem and progenitor cell populations in the bone marrow of male mice treated with vehicle, E2, or 27HC daily for 6 days (a total of 3-4 mice/treatment from 2 independent experiments). (E) Plasma 27HC levels in male mice treated with vehicle or 27HC daily for 6 days (a total of 5 mice/treatment from 5 independent experiments). (F) BrdU incorporation into hematopoietic stem and progenitor cells in the spleens of male mice treated with 27HC daily for 6 days. The mice received BrdU for the last 3 days (a total of 5 mice/treatment from 4 independent experiments). Statistical significance was assessed using 1-way ANOVA with Šídák's multiple comparisons tests, with the exception $\mathbf{E}$, where we used Welch's test $\left({ }^{\ddagger} P<0.01\right)$ and $\mathbf{F}$, where we used 2-tailed unpaired Student's $t$ tests using the false discovery rate (FDR) method to correct for multiple comparisons $\left({ }^{*} P<0.05,{ }^{\ddagger} P<0.01,{ }^{\#} P<0.001\right)$. See Methods for details. All data represent mean \pm SD.

dependent manner. 27HC levels increase in HSPCs during pregnancy and promote EMH. Cyp27a1 deficiency prevented the increase in 27HC levels, impairing HSC mobilization and EMH during pregnancy, but not affecting normal bone marrow hematopoiesis or EMH in response to bleeding or G-CSF treatment. Distinct hematopoietic stresses thus induce EMH through distinct mechanisms. $27 \mathrm{HC}$ acts in concert with estradiol to promote $\mathrm{EMH}$ during pregnancy by regulating ERa function in HSCs.

\section{Results}

Estradiol induces HSC division but not mobilization. The increases in HSC division, HSC mobilization, and EMH during pregnancy require ER $\alpha$ in HSCs and HPCs (2). Administration of E2 promotes HSC division in the bone marrow (2), but it is unknown whether estrogen promotes HSC mobilization. To test whether E2 promotes HSC mobilization, we treated male mice with E2 daily $(100 \mu \mathrm{g} / \mathrm{kg} / \mathrm{d})$ for 6 days and analyzed the bone marrow and spleen (Figure 1, A and B). As we published previously (2), E2 administration did not affect the number of $\mathrm{CD} 150^{+} \mathrm{CD} 48^{-/ 10} \mathrm{CD} 34^{-/ 10} \mathrm{CD} 135$ - Lineage-Sca- ${ }^{+} \mathrm{c}-\mathrm{kit}^{+}$ HSCs in the bone marrow or bone marrow cellularity (Figure 1A), but it did significantly increase BrdU incorporation by HSCs (Figure 1C). E2 treatment did not significantly affect BrdU incorporation by other primitive progenitors in the bone marrow, or by unfractionated whole bone marrow (WBM) cells, with the exception of HPC-1 cells, which exhibited decreased BrdU incorporation (Figure 1C).

E2 treatment significantly reduced the numbers of $\mathrm{CD}_{150}$ $\mathrm{CD} 48^{-/ /}$Lineage-Sca- $1^{+} \mathrm{c}-\mathrm{kit}^{+}$multipotent progenitors (MPPs), CD150-CD $48^{+}$Lineage-Sca- $1^{+} \mathrm{c}-\mathrm{kit}^{+}$hematopoietic progenitor cells (HPC-1), CD150 ${ }^{+} \mathrm{CD} 48^{+}$Lineage ${ }^{-} \mathrm{Sca}-1^{+}{ }^{+}$-kit ${ }^{+}$HPC-2 progenitors (17), and common myeloid progenitors (CMPs) (18) in the bone marrow (Figure 1A). Tamoxifen, a synthetic estrogen receptor modulator, induces apoptosis in primitive hematopoietic progeni- 
tors (19). E2 treatment also induced apoptosis in HPCs, as indicated by increased annexin $\mathrm{V}$ binding to exteriorized phosphatidylserine (Figure 1D). This may contribute to the declines in the numbers of HPCs and other restricted progenitors in E2-treated mice.

Despite increasing HSC proliferation in the bone marrow, E2 treatment did not significantly increase the numbers of HSCs or progenitors in the spleen, or overall spleen cellularity (Figure 1B). Therefore, E2 does not promote HSC mobilization, and its actions do not explain the ER $\alpha$-dependent increase in EMH observed in pregnant mice (2).

27HC induces HSC mobilization. To test whether another endogenous ER $\alpha$ ligand can induce HSC mobilization, we treated male mice with $27 \mathrm{HC}$ daily $(20 \mathrm{mg} / \mathrm{kg} / \mathrm{d}$ ) for 6 days (Figure $1, \mathrm{~A}$ and B). This significantly increased plasma $27 \mathrm{HC}$ levels (Figure 1E). 27HC treatment did not significantly affect the numbers of HSCs or other hematopoietic progenitors in the bone marrow or total bone marrow cellularity (Figure 1A). 27HC treatment also did not significantly affect BrdU incorporation by HSCs, MPPs, HPC-1 cells, HPC-2 cells, or unfractionated cells in the bone marrow (Figure 1C) or spleen (Figure 1F). In contrast to E2 treatment, 27HC treatment did not induce apoptosis in stem or progenitor cells in the bone marrow (Figure 1D).

However, 27HC treatment did significantly increase the numbers of HSCs and other hematopoietic progenitors in the spleen, as well as overall spleen cellularity (Figure 1B). Sections through the spleen showed increased EMH in 27HC-treated mice as compared with vehicle-treated controls (Figure 2A). 27HC thus promotes HSC mobilization and EMH.

27HC-induced HSC mobilization is not mediated by G-CSF signaling. Mice with defects in cholesterol efflux exhibit increased HSPC mobilization associated with increased serum G-CSF levels (15). Cholesterol efflux defects increase intracellular cholesterol, and potentially $27 \mathrm{HC}$; therefore, we tested whether HSC mobilization by $27 \mathrm{HC}$ depends upon G-CSF. In the bone marrow, G-CSF-treated mice had more HPC-2 cells and fewer MPPs and CMPs as compared with 27HC-treated mice (Figure 1A). In the spleen, G-CSF-treated mice had more HPC-2 cells and fewer megakaryocyte-erythroid progenitors (MEPs) as compared with 27HC-treated mice (Figure 1B). To directly test whether G-CSF is required for $27 \mathrm{HC}$-induced HSC mobilization, we treated G-CSFdeficient $\left(\mathrm{Csf3}^{-/-}\right)$mice with 27HC. 27HC treatment did not significantly affect the numbers of HSCs, MPPs, HPC-1 cells, HPC2 cells, CMPs, granulocyte-macrophage progenitors (GMPs), or MEPs in the bone marrow of either $\mathrm{Csf3}^{-/-}$or $\mathrm{Csf3^{+/+ }}$ mice (Figure 2B). $27 \mathrm{HC}$ treatment did significantly increase the numbers of HSCs, MPPs, and HPC-2 cells in the spleens of $\mathrm{Csf3}^{-/-}$and $\mathrm{Csf3}^{+/+}$ mice (Figure 2C). G-CSF deficiency did not affect the magnitude of the increase in splenic HSCs or MPPs in response to $27 \mathrm{HC}$. Together, 27HC and G-CSF additively increased the numbers of colony-forming progenitors in the blood significantly beyond either agent alone (Figure 2D). Therefore, 27HC does not require G-CSF to mobilize HSCs or MPPs to the spleen. 27HC and G-CSF likely act through distinct mechanisms.

27HC-induced HSC mobilization is mediated by ER $\alpha$. To test whether the effect of $27 \mathrm{HC}$ on hematopoietic cells is mediated by $\mathrm{ER} \alpha$, we conditionally deleted Esr1 (the gene that encodes ER $\alpha$ ) from hematopoietic cells. We treated male Vav1-icre; Esr $1^{f / f l}$ mice or male littermate controls with $27 \mathrm{HC}$. As expected, $27 \mathrm{HC}$ treatment or Esr1 deletion did not significantly affect bone marrow cellularity or the numbers of stem/progenitor cells in the bone marrow (Figure 2E). 27HC treatment did significantly increase the numbers of HSCs, MPPs, HPC-1 cells, and HPC-2 cells in the spleens of control mice but not Vav1-icre; Esr $1^{f / f l}$ littermates (Figure 2F). The inability of 27HC to promote the mobilization of Esr1-deficient HSCs or hematopoietic progenitors indicates that $27 \mathrm{HC}$ mobilizes these cells by modulating ER $\alpha$ function in hematopoietic cells.

To test whether $27 \mathrm{HC}$ acts directly on HSCs, we competitively transplanted $1 \times 10^{6} \mathrm{CD} 45.2^{+}$Vav1-icre; Esr $1^{f / f l}$ donor bone marrow cells along with $1 \times 10^{6} \mathrm{CD} 45.1^{+}$WT competitor bone marrow cells into irradiated CD $45.1^{+}$male mice. Four months later we treated the recipient mice with either vehicle or $27 \mathrm{HC}$ for 6 days and analyzed the frequencies of donor-derived cells in the bone marrow and spleen. Total donor cell reconstitution, and reconstitution in the myeloid, B, and $\mathrm{T}$ cell lineages, in the bone marrow, spleen, and blood did not significantly differ between 27HC-treated and vehicle-treated mice (Figure 3, A-F). As expected, 27HC treatment did not significantly affect the frequencies of donor-derived HSCs, MPPs, HPC- 1 cells, HPC-2 cells, CMPs, GMPs, or MEPs in the bone marrow (Figure 3B). However, 27HC-treated mice had significantly lower frequencies of donor-derived (Esr1-deficient) HSCs and HPC-2 cells in the spleen as compared with vehicletreated mice (Figure 3F). This indicates that Esr1-deficient HSCs and primitive progenitors were at a disadvantage compared with WT cells in the same mice for mobilization in response to $27 \mathrm{HC}$. $\mathrm{ER} \alpha$ thus acts cell-autonomously within HSCs and other primitive progenitors to promote mobilization in response to $27 \mathrm{HC}$.

Normal hematopoiesis in Cyp27a1-deficient mice. To assess the physiological role of $27 \mathrm{HC}$ in hematopoiesis, we analyzed Cyp27a1 1 - mice, which had greatly reduced plasma $27 \mathrm{HC}$ levels (Figure 4A). Cyp27a1 deficiency did not affect WBM or spleen cellularity or the numbers of HSCs, MPPs, HPC-1 cells, HPC-2 cells, CMPs, GMPs, or MEPs in the bone marrow (Figure 4D) or spleen (Figure 4E). Cyp27a1 deficiency also did not affect the frequency of colony-forming progenitors in the blood (Figure 4B). These data included both male and female Cyp27a1 ${ }^{-1-}$ mice. $27 \mathrm{HC}$ was thus dispensable for steady-state hematopoiesis. Consistent with the observation that HSCs divide more frequently in female as compared with male mice (2), HSCs in female mice incorporated significantly more BrdU as compared with HSCs in male mice, and Cyp27a1 deficiency did not affect the rate of BrdU incorporation (Figure $4 \mathrm{C}$ ). This suggests that E2, but not 27HC, promotes increased HSC division.

CYP27A1 is required for HSC mobilization during pregnancy. We next assessed the function of $27 \mathrm{HC}$ in the activation of hematopoiesis during pregnancy by analyzing pregnant dams on day 14.5 of gestation. Pregnant WT mice exhibited modest but significant increases in the numbers of HSCs, HPC-2 cells, and CMPs in the bone marrow as compared with non-pregnant female WT mice (Figure 5A). Pregnant Cyp27a1 $1 /-$ mice did not significantly differ from pregnant WT mice in terms of bone marrow cellularity or the numbers of HSCs or other progenitors in the bone marrow (Figure $5 \mathrm{~A})$. In contrast, pregnant WT mice exhibited substantial increases in the numbers of HSCs, MPPs, HPC- 1 cells, HPC-2 cells, CMPs, GMPs, MEPs, and erythroid lineage cells in the spleen as com- 
A

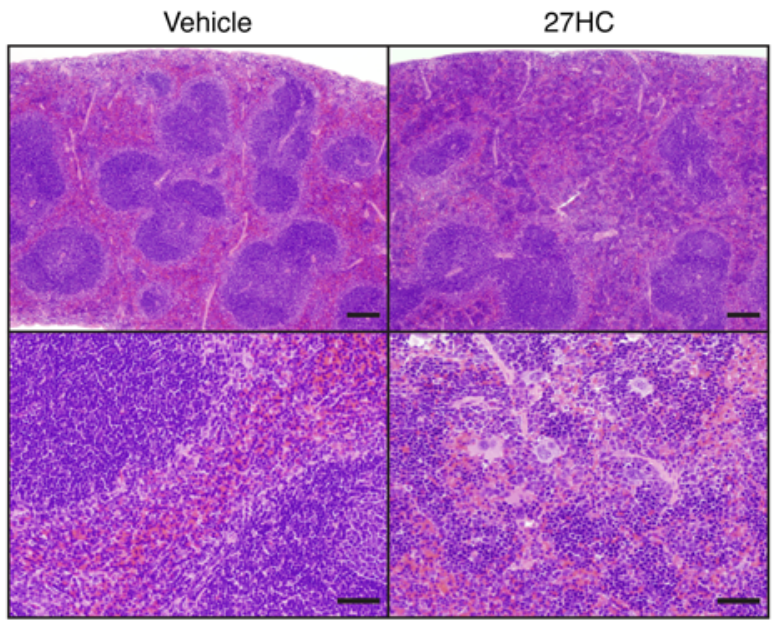

B

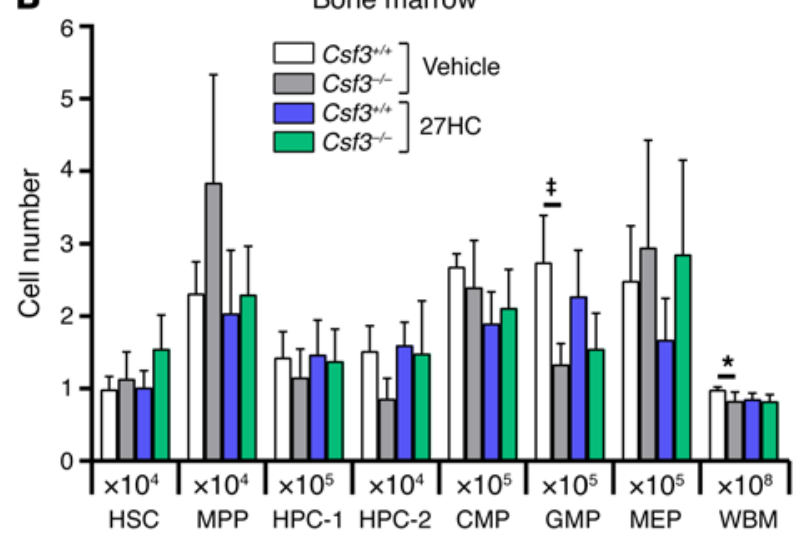

C

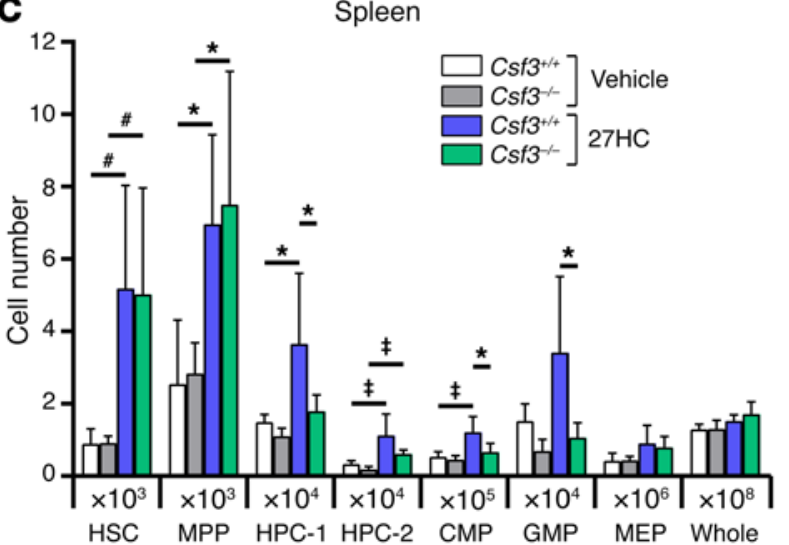

D

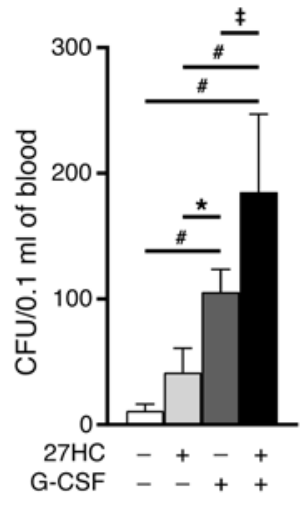

E

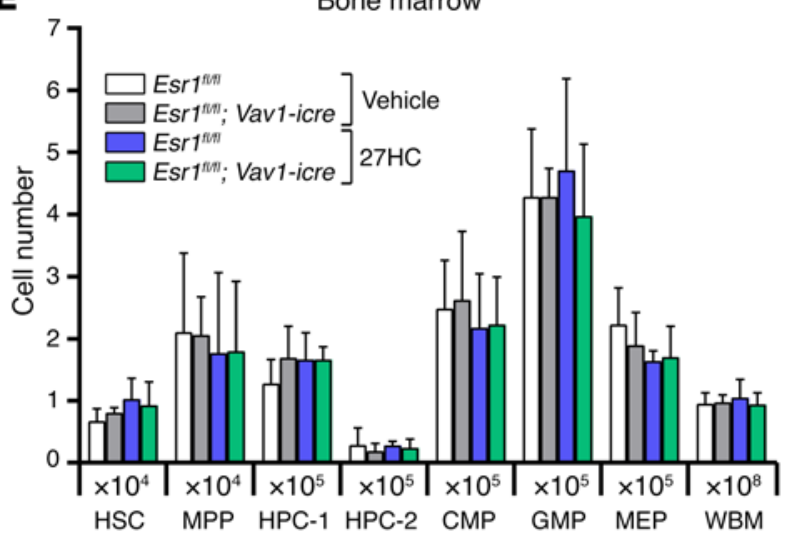

$\mathbf{F}$

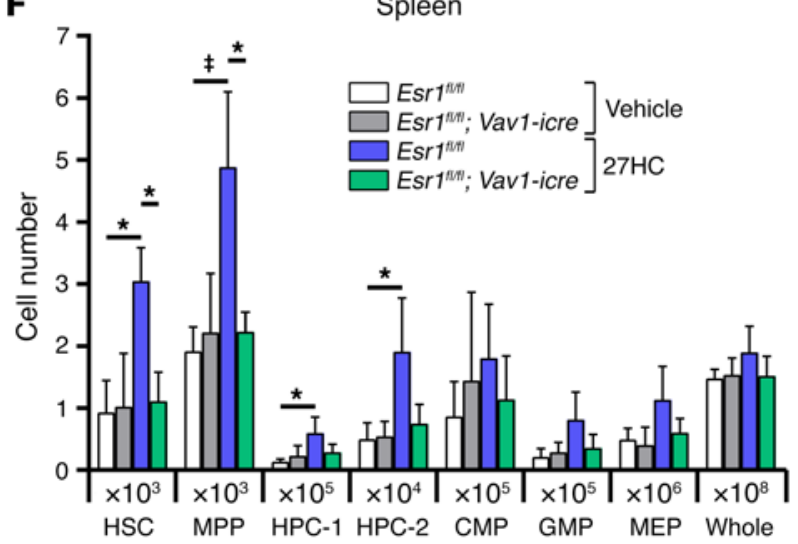

Figure 2. 27HC-induced mobilization of hematopoietic stem and progenitor cells requires Esr1 but not Csf3. (A) Representative spleen sections stained with $\mathrm{H} \& \mathrm{E}$ from mice treated with vehicle (left) or 27HC (right) daily for 6 days. Lower-magnification (top) and higher-magnification (bottom) images are shown. Scale bars: $200 \mu \mathrm{m}$ (top panels); $50 \mu \mathrm{m}$ (bottom panels). (B and C) The numbers of hematopoietic stem and progenitor cells in the bone marrow (femurs and tibias; B) and spleen (C) of $\mathrm{Csf}^{+/+}$or $\left[s f 3^{-/-}\right.$mice treated with vehicle or $27 \mathrm{HC}$ daily for 6 days (a total of 5 mice/treatment from 4 independent experiments). (D) Numbers of CFU in the blood of mice treated with vehicle, 27HC, G-CSF, or a combination of 27HC and G-CSF daily for 6 days (a total of 4-6 mice/treatment from 6 independent experiments). (E and F) Numbers of hematopoietic stem and progenitor cells in the bone marrow (femurs and tibias; E) and spleen (F) of Vav1-icre; Esr ffl/fl mice or Esr $f^{f / / f l}$ controls treated with vehicle or 27HC daily for 6 days (a total of 3 mice/treatment from 3 independent experiments). Statistical significance was assessed using 1-way ANOVA with Šídák's multiple comparisons tests $\left({ }^{*} P<0.05\right.$, $\left.{ }^{\ddagger} P<0.01,{ }^{*} P<0.001\right)$. All data represent mean \pm SD.

pared with non-pregnant female WT mice (Figure 5B). Cyp27a1 deficiency largely blocked these increases in the numbers of stem and progenitor cells in the spleens of pregnant mice (Figure $5 \mathrm{~B}$ ). Pregnant Cyp27a1 ${ }^{-/-}$mice did not significantly differ from non- pregnant WT mice in terms of spleen cellularity or the numbers of HSCs or MPPs in the spleen (Figure 5B). Consistent with these results, pregnant WT mice had more colony-forming progenitors in their blood as compared with non-pregnant WT mice, but 
A

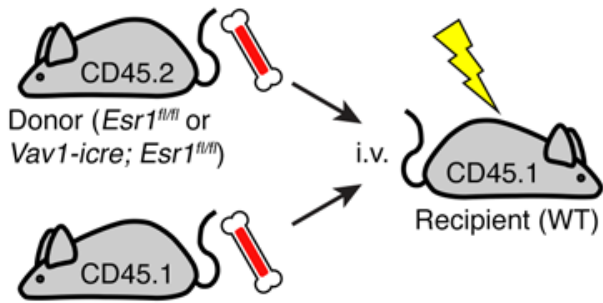

Competitor (WT)

B

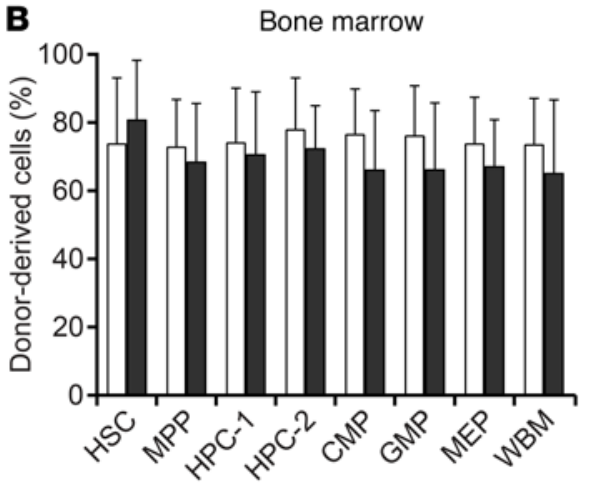

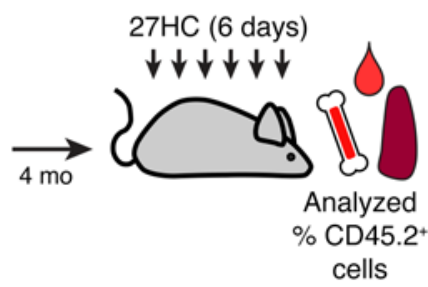

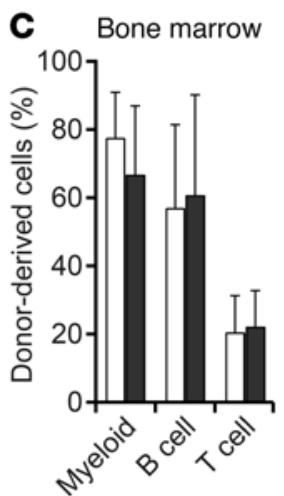

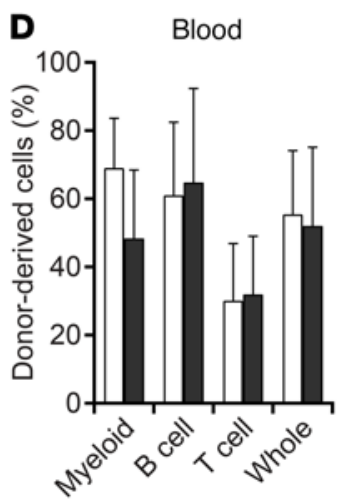

Figure 3. 27HC acts directly on HSCs to promote ER $\alpha$-dependent mobilization. (A) Transplantation of $1 \times 10^{6}$ donor (CD45.2 $2^{+}$) bone marrow cells from Vav1-icre; Esr $7^{f / f l}$ mice along with $1 \times 10^{6}$ competing recipient (CD45.1 $1^{+}$) bone marrow cells into irradiated recipient (CD45.1 $1^{+}$mice. (B-F) Data represent the percentages of donor-derived cells in the indicated populations from the bone marrow (B and $\mathbf{C}$ ), blood (D), and spleen (E and F) of recipient mice treated with vehicle or $27 \mathrm{HC}$ daily for 6 days beginning at 4 months after transplantation (a total of 4 mice/treatment from 2 independent experiments). Statistical significance was assessed using 2-tailed unpaired Student's $t$ tests using the FDR method to correct for multiple comparisons $\left({ }^{*} P<0.05\right)$. All data represent mean \pm SD.
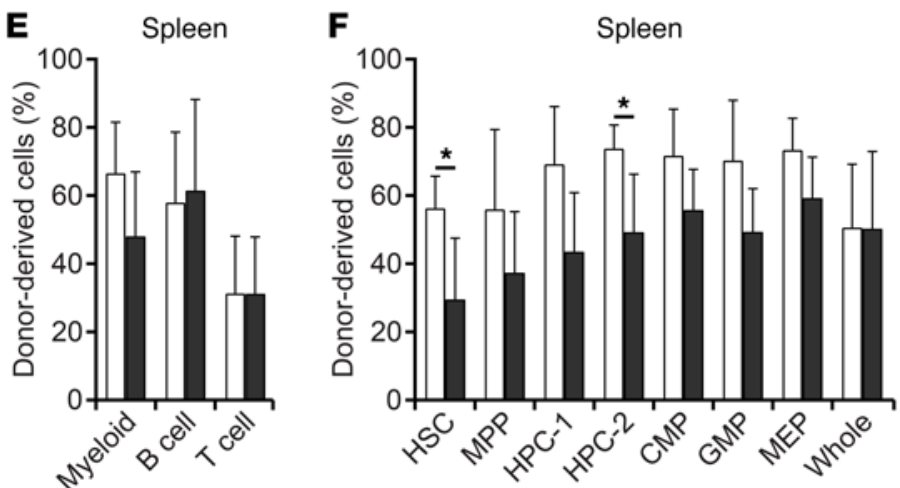

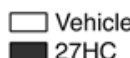

Cyp27a1 deficiency blocked most of this increase during pregnancy (Figure 5C). Cyp27a1 was thus required for HSC and progenitor mobilization, as well as EMH during pregnancy. Taken together, the data indicate that the CYP27A1 product 27HC promotes HSC and progenitor mobilization as well as $\mathrm{EMH}$ during pregnancy.

The rates of BrdU incorporation by HSCs in the bone marrow and spleen, as well as unfractionated spleen cells, significantly increased in pregnant as compared with non-pregnant WT mice, consistent with the induction of EMH during pregnancy (Figure $5 \mathrm{D})$. These increases in BrdU incorporation were not affected by Cyp27a1 deficiency in pregnant mice (Figure 5D). This indicates that $27 \mathrm{HC}$ does not affect the rate of HSC division in the bone marrow or spleen, just HSC mobilization.

CYP27A1 is not required for EMH in response to bleeding or G-CSF treatment. To test whether Cyp27a1 also regulates HSC mobilization/EMH in response to other hematopoietic stimuli, we investigated hematopoiesis in $C y p 27 a 1^{-1-}$ mice after repeated bleeding or G-CSF treatment. As expected $(3,20)$, repeated bleeding induced $\mathrm{EMH}$, significantly increasing the numbers of HSCs, other hematopoietic progenitors, and overall spleen cellularity (Figure 6B). Cyp27a1 deficiency did not affect the induction of EMH by repeated bleeding: we observed no significant differ- ences between Cyp27a1 ${ }^{-/-}$and littermate control mice in terms of numbers of HSCs, hematopoietic progenitors, or overall cellularity in the spleen or bone marrow after repeated bleeding (Figures 6, A and B). G-CSF treatment also induced EMH, significantly increasing the numbers of HSCs, other hematopoietic progenitors, and overall spleen cellularity (Figure 6D). Cyp27a1 deficiency did not affect the induction of EMH by G-CSF: we observed no significant differences between $C y p 27 a 1^{-/-}$and littermate control mice in terms of numbers of HSCs, hematopoietic progenitors, or overall cellularity in the spleen or bone marrow after G-CSF treatment (Figures 6, C and D). Distinct hematopoietic stresses thus induce EMH through distinct mechanisms. While 27HC synthesized by CYP27A1 is required for the induction of EMH during pregnancy, it is not required for EMH induction in response to bleeding or G-CSF treatment.

27 HC levels increase in hematopoietic progenitors during pregnancy. Plasma total cholesterol levels increase during pregnancy in humans (21), and plasma $27 \mathrm{HC}$ levels increase as total cholesterol levels increase (6). We did not detect an increase in $27 \mathrm{HC}$ levels in the plasma or liver during pregnancy in mice (data not shown). However, we did observe significant increases in Cyp27a1 expression in HSCs and HPC-1 cells (Figure 6E), as well as 27HC levels 
A

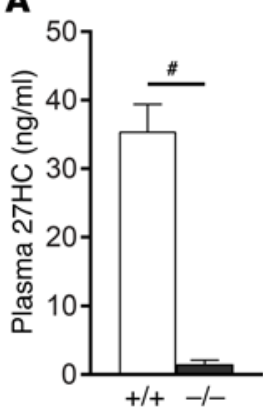

B
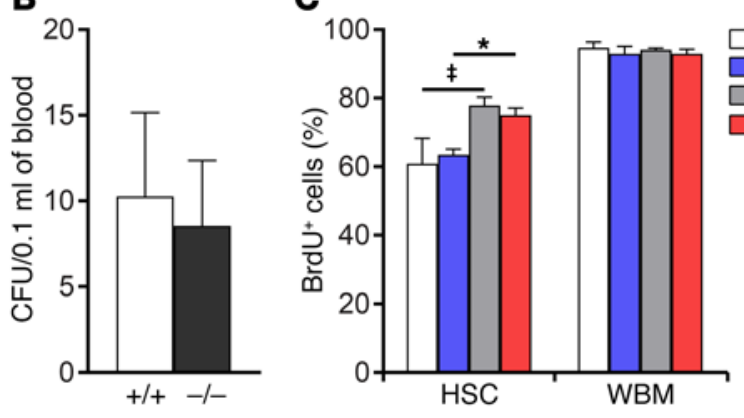

D

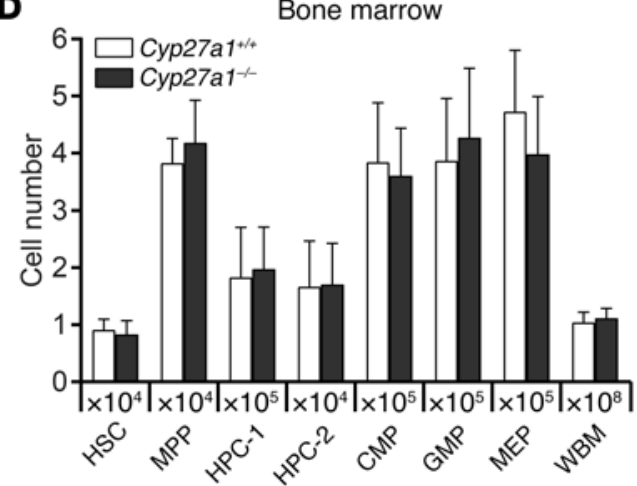

E

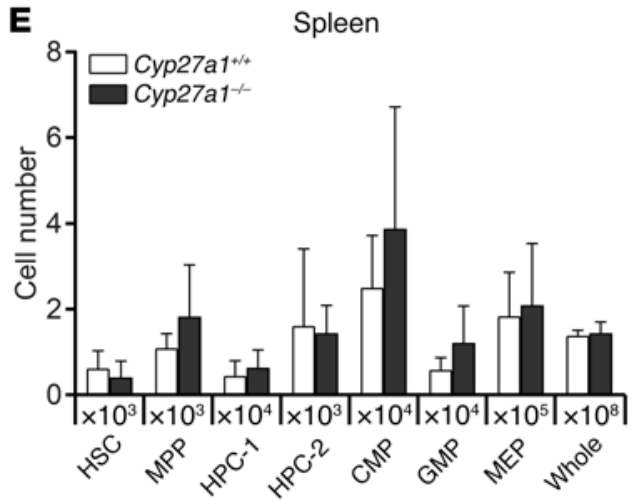

Figure 4. Cyp27a1 deficiency does not affect hematopoiesis under steady-state conditions. (A) Plasma 27HC levels in WT and Cyp27a1 1 - mice (a total of 3 mice/treatment from 3 independent experiments). (B) Colony-forming progenitors in the blood of WT and Cyp27a1 ${ }^{-/-}$mice (a total of 6 mice/genotype from 3 independent experiments). (C) Incorporation of a 7-day pulse of BrdU by HSCs or whole bone marrow cells from WT or Cyp27a1 1 male or female mice (a total of 3 mice/treatment from 3 independent experiments). ( $\mathbf{D}$ and $\mathbf{E})$ The numbers of hematopoietic stem and progenitor cells in the bone marrow (femurs and tibias; $\mathbf{D}$ ) or spleen (E) of 3-month-old Cyp27a $11^{-/-}$and littermate control mice (a total of 5 mice/genotype from 4 independent experiments). Statistical significance was assessed using 2-tailed unpaired Student's $t$ tests using the FDR method to correct for multiple comparisons, except in C, where we used 1-way ANOVA with Šídák's multiple comparisons tests ${ }^{*} P<0.05$, ${ }^{\ddagger} P<0.01,{ }^{\#} P<0.001$ ). All data represent mean \pm SD.

in Lineage-negative bone marrow cells during pregnancy (Figure $6 \mathrm{~F})$. These data suggest that $27 \mathrm{HC}$ synthesis increases in HSCs and other hematopoietic progenitors during pregnancy, consistent with our data indicating that the $27 \mathrm{HC}$ receptor $\mathrm{ER} \alpha$ acts cellautonomously to promote mobilization during pregnancy.

CXCL12/CXCR4 signaling is necessary for the retention of HSCs in the bone marrow, and inhibition of CXCL12/CXCR4 signaling causes HSC mobilization (22-24). Hypercholesterolemia promotes HSPC mobilization by dysregulating CXCL12/CXCR4 signaling (11), and ER $\alpha$ represses cxcr $4 b$ expression in zebrafish (25). Since plasma $27 \mathrm{HC}$ levels increase as total cholesterol levels increase (6) and increased 27HC/ER $\alpha$ signaling promotes HSC mobilization (Figures 2 and 3), we hypothesized that hypercholesterolemia promotes HSC mobilization through a $27 \mathrm{HC} /$ ER $\alpha$-mediated reduction in Cxcr4 expression. Consistent with this hypothesis, pregnant mice exhibited a significant reduction in Cxcr4 expression in HSCs, and this reduction was rescued by Cyp27a1 deficiency (Figure 6G). Cxcr4 expression in HSCs from pregnant Cyp27a1 $1 /$ mice was significantly higher than in HSCs from pregnant WT mice and statistically indistinguishable from that in non-pregnant WT mice. Cyp27a1 deficiency did not affect Cxcr4 expression in HSCs from non-pregnant mice (Figure 6H). conformation (26-29). For example, Wardell et al. tested 6 different ER ligands and observed different gene expression patterns regulated by different ER-ligand complexes (29). Unliganded ER $\alpha$ can also bind to chromatin and regulate gene expression (30). $27 \mathrm{HC}$ induces a unique conformational change in ER that is different from that mediated by E2 and other ER ligands (31). Different ER-ligand complexes also engage functionally distinct coregulators (32). Thus, different ER ligands have different effects on the conformation and function of ER, leading to different effects on gene expression. This may explain why E2 and 27HC have distinct effects on HSCs even though both act through ER $\alpha$.

In addition to $27 \mathrm{HC}$, bile acids are also synthesized from cholesterol, and can serve as chemical chaperones that regulate proteostasis and fetal liver HSC frequency (33). However, the effects of 27HC administration or Cyp27a1 deletion on HSC mobilization cannot be explained solely by changes in bile acid synthesis, because the effect of 27HC on HSCs required ER $\alpha$ expression by HSCs (Figures 2 and 3). ER $\alpha$ is a receptor for $27 \mathrm{HC}$ but not bile acids. Had 27HC promoted mobilization through changes in bile acid biosynthesis, rather than by regulating ER $\alpha$ function in HSCs, $27 \mathrm{HC}$ should have equally promoted the mobilization of both WT and ER $\alpha$-deficient HSCs. Since ER $\alpha$-deficient HSCs were not mobi- 

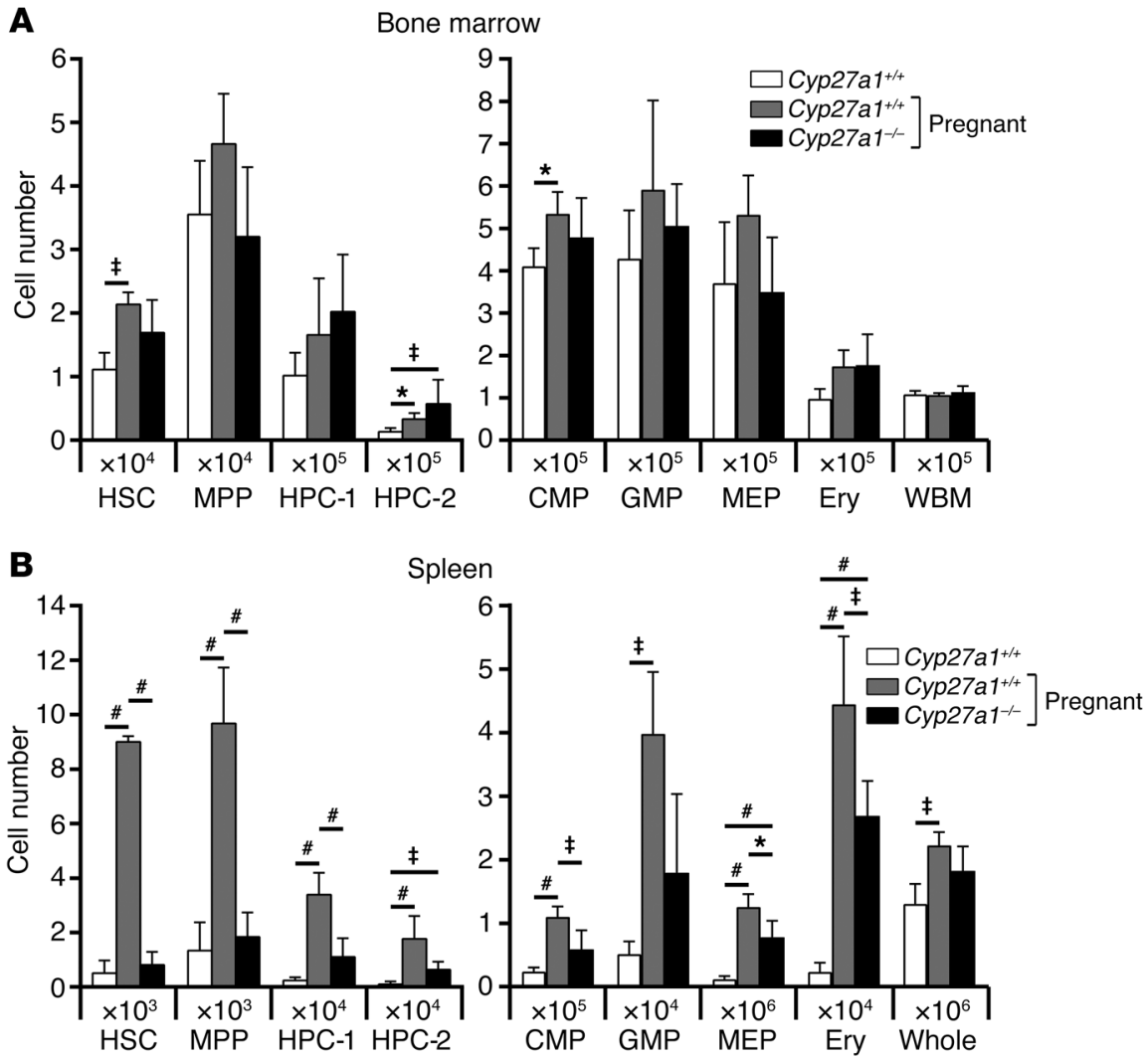

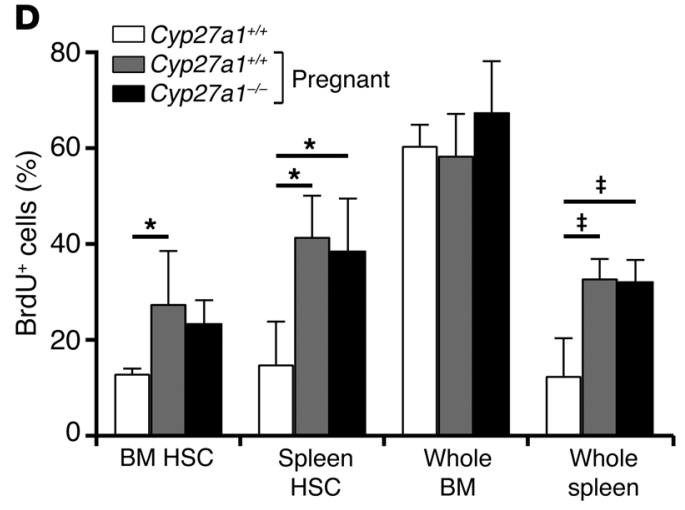

Figure 5. Pregnancy-induced HSC mobilization requires Cyp27a1, the enzyme that produces 27HC. (A and B) The numbers of hematopoietic stem and progenitor cells in the bone marrow (femurs and tibias; A) and spleen (B) of WT nonpregnant mice, WT pregnant mice, and Cyp27a1 ${ }^{-1}$ pregnant mice (a total of 4-5 mice/treatment from 5 independent experiments). Pregnant mice were analyzed on day 14.5 of gestation. (C) CFU in the blood of WT non-pregnant mice, WT pregnant mice, and Cyp27a $1 /$ pregnant mice (a total of 7 mice/treatment from 8 independent experiments). (D) Incorporation of a 24 -hour pulse of BrdU by HSCs or unfractionated cells from the bone marrow or spleen of WT non-pregnant mice, WT pregnant mice, and Cyp27a1-/ pregnant mice (a total of 3-5 mice/treatment from 4 independent experiments). Statistical significance was assessed using 1-way ANOVA with Šídák's multiple comparisons tests $\left({ }^{*} P<0.05,{ }^{\ddagger} P<0.01\right.$, $\left.{ }^{\#} P<0.001\right)$. All data represent mean \pm SD.

We observed that 27HC and G-CSF treatment had additive effects on HSPC mobilization (Figure 2D). This raises the possibility that treatments that enhance 27HC/ER $\alpha$ signaling could have beneficial effects on clinical HSC mobilization.

\section{Methods}

Mice. The mice used in this study were Esr $1^{f / f l}$ (36), Vav1-icre (37), Cyp27a1 $1^{-/}$(7), and Csf3 $3^{-1-}$ (38). These mice were all backcrossed for at least 8 generations onto a C57BL background. WT mice were young adult C57BL/KaCD45.2:Thy1.1 mice (8-12 weeks of age) unless otherwise noted. C57BL/Ka-CD45.1:Thy1.2 mice were used as recipients in transplantation experiments. Mice were injected subcutaneously with $100 \mu \mathrm{g} / \mathrm{kg} / \mathrm{d}$ E2 (Sigma-Aldrich) in corn oil (Sigma-Aldrich), $20 \mathrm{mg} / \mathrm{kg} / \mathrm{d} 27 \mathrm{HC}$ (Avanti Polar Lipids) in 30\% 2-hydroxypropyl-

lized as efficiently as WT HSCs in the same mice (Figure 3F), 27HC promotes mobilization by regulating ER $\alpha$ function. Nonetheless, it is possible that in addition to regulating $\mathrm{ER} \alpha$ function, $27 \mathrm{HC}$ also influences HSCs through effects on bile acid biosynthesis.

Our results offer a potential explanation for the long-standing observation that increased cholesterol levels are associated with increased HSPC mobilization in mice and humans (11-16). 27HC levels increase as cholesterol levels increase (6). Several studies have reported a correlation between cholesterol levels and HSPC numbers in human blood (34). Patients with hypercholesterolemia mobilize larger numbers of $\mathrm{CD} 4^{+}$HSPCs following treatment with cyclophosphamide and G-CSF as compared with patients with lower cholesterol levels (14). Moreover, plasma cholesterol levels increase in humans during pregnancy (35). The ability of elevated cholesterol levels to promote HSPC mobilization may be mediated by increases in $27 \mathrm{HC}$ production. $\beta$-cyclodextrin (Sigma-Aldrich), or $250 \mu \mathrm{g} / \mathrm{kg} / \mathrm{d}$ G-CSF (Amgen) in PBS (Gibco). Male mice were used in most of the experiments, unless otherwise noted. Female mice were used in pregnancy experiments. All mice used in this study were housed in the Animal Resource Center at the University of Texas Southwestern Medical Center.

Flow cytometry and cell isolation. Bone marrow cells were isolated by flushing the femurs and tibiae or by crushing the femurs, tibiae, pelvic bones, and vertebrae with a mortar and pestle in HBSS without calcium or magnesium (Gibco), supplemented with $1 \%$ heat-inactivated bovine serum (Gibco) and filtered through a $45-\mu$ m nylon screen (Sefar America). Spleens were dissociated by crushing, followed by gentle trituration and filtering through a $40-\mu \mathrm{m}$ cell strainer (Fisher Scientific). Cell number and viability were assessed by a Vi-CELL cell viability analyzer (Beckman Coulter) or by counting with a hemocytometer. For isolation of HSCs and progenitors, a mixture of antibodies against CD2, CD3, CD5, CD8 $\alpha$, B220, Gr-1, and Ter119 was used to stain lineage mark- 
A

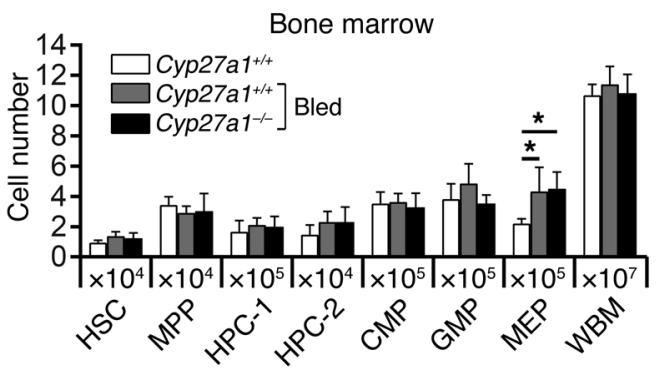

C

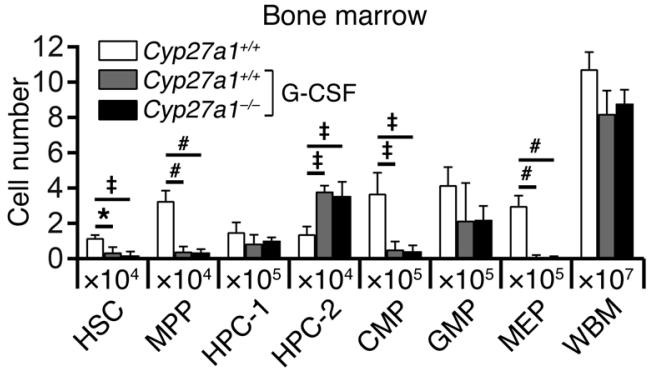

E

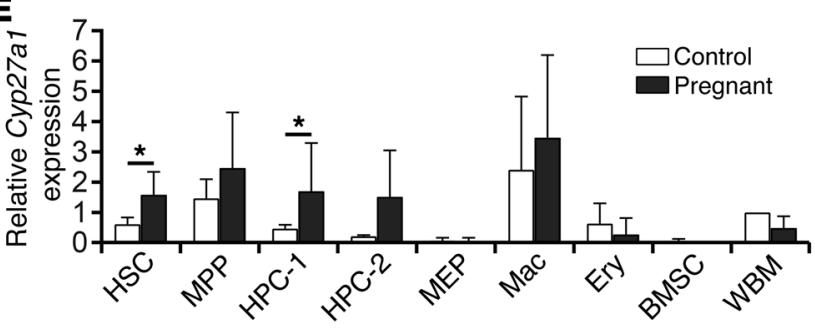

B

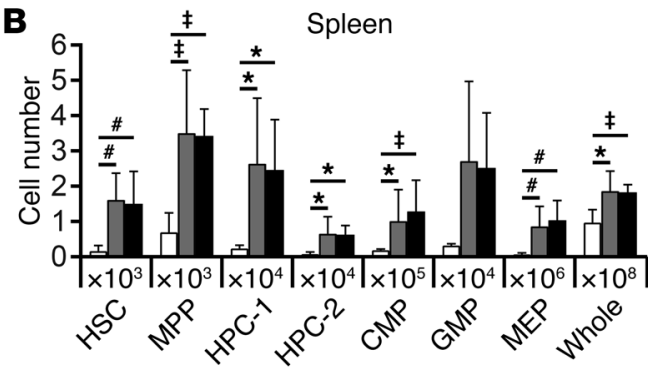

D

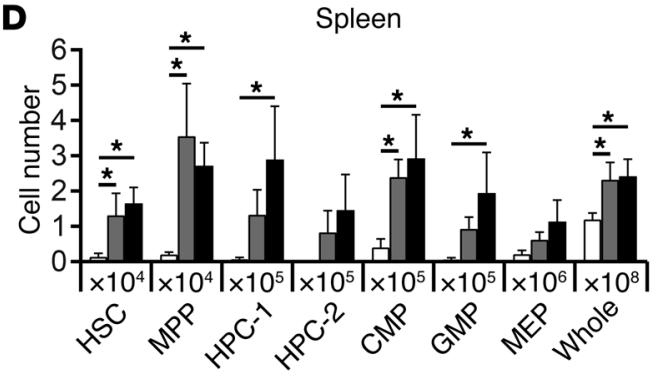

F

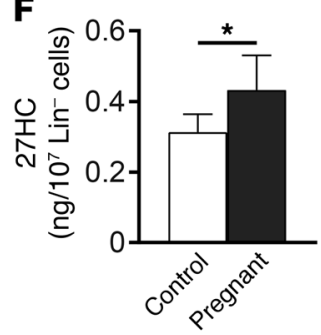

G

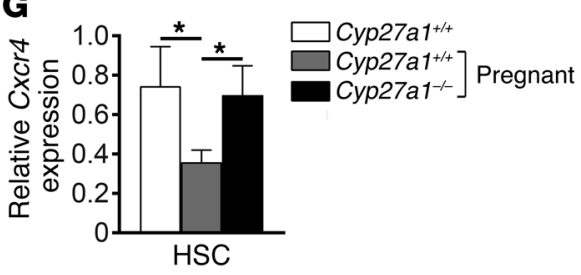

H

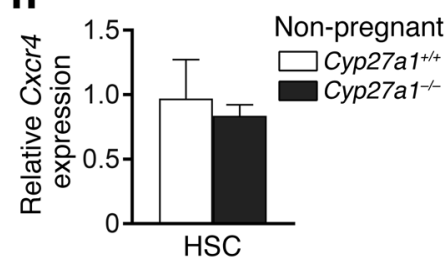

Figure 6. Pregnancy increases Cyp27a1 expression and 27HC levels in primitive hematopoietic stem and progenitor cells. (A and B) Cyp27a1 deficiency did not affect the numbers of hematopoietic stem and progenitor cells in the bone marrow (femurs and tibias; $\mathbf{A}$ ) or spleen (B) of mice that had been repeatedly bled (a total of 5-6 mice/treatment from 3 independent experiments). (C and D) Cyp27a1 deficiency did not affect the numbers of hematopoietic stem and progenitor cells in the bone marrow (femurs and tibias; C) or spleen (D) of mice treated with G-CSF for 6 days (a total of 3 mice/treatment from 2 independent experiments). (E, $\mathbf{G}$, and $\mathbf{H})$ Cyp27a1 (E) or $C x c r 4$ ( $\mathbf{G}$ and $\mathbf{H}$ ) transcript levels were quantified by qRT-PCR in the indicated bone marrow cell populations (a total of 3-5 mice from 3-4 independent experiments). Expression values were normalized to beta-Actin and presented relative to those in control WBM cells. (F) Intracellular 27HC levels in $10^{7}$ lineage-depleted bone marrow cells from non-pregnant and pregnant WT mice (a total of 5 mice/ treatment from 4 independent experiments). Statistical significance was assessed using 1-way ANOVA with Šídák's multiple comparisons tests, with the exception of $\mathbf{E}, \mathbf{F}$, and $\mathbf{H}$, where we used 2-tailed unpaired Student's $t$ tests using the FDR method to correct for multiple comparisons $\left({ }^{*} P<0.05\right.$, ${ }^{\ddagger} P<0.01$, $\left.{ }^{\#} P<0.001\right)$. All data represent mean $\pm \mathrm{SD}$.

ers. Bone marrow cells were incubated with biotinylated antibodies against lineage markers, followed by anti-biotin microbeads, and Lineage $^{+}$cells were depleted using an autoMACS Pro Separator (Miltenyi Biotec). Non-viable cells were excluded during flow cytometry by staining with 4',6-diamidino-2-phenylindole (Sigma-Aldrich) or propidium iodide (Molecular Probes). Apoptotic cells were identified using APCconjugated anti-annexin V antibody (BD Biosciences). Antibodies (and clones) used in this study were anti-CD2 (RM2-5), anti-CD3 (17A2), anti-CD5 (clone 53-7.3), anti-CD8 $\alpha$ (clone 53-6.7), anti-CD11b/Mac-1 (M1/70), anti-CD16/32 (clone 93), anti-CD31 (MEC13.3), anti-CD34 (RAM34), anti-CD45 (30-F11), anti-CD45.1 (A20), anti-CD45.2 (clone
104), anti-CD45R/B220 (RA3-6B2), anti-CD48 (HM48-1), anti-CD71 (R17217), anti-CD105 (MJ7/18), anti-CD117/c-kit (2B8), anti-CD135/ FLT3 (A2F10), anti-CD140a/PDGFR $\alpha$ (APA5), anti-CD150 (TC1512F12.2), anti-CD184/CXCR4 (2B11), anti-F4/80 (BM8), anti-Sca-1 (D7 and E13-161.7), anti-Gr-1 (RB6-8C5), and anti-Ter119 (TER-119). Antibodies and streptavidin were conjugated to one of the following dyes or to biotin: Pacific Blue, Brilliant Violet 421, Brilliant Violet 510, FITC, PerCP-Cy5.5, PE, PE-CF594, PE-Cy5, PE-Cy7, APC, Alexa Fluor 700, APC-eFluor 780. All antibodies were purchased from BioLegend, eBioscience, BD Biosciences, or Tonbo Biosciences. Data acquisition and cell sorting were performed using a FACSAria II or FACSCanto 
II (BD Biosciences), and data were analyzed using FlowJo software. The marker combinations used to identify the hematopoietic stem and progenitor cell populations examined in this study were: HSC: $\mathrm{CD} 15 \mathrm{O}^{+} \mathrm{CD} 48^{-/ / \mathrm{lo}} \mathrm{CD} 34^{-/ \mathrm{lo}} \mathrm{CD} 135^{-}$Lineage-Sca- ${ }^{+} \mathrm{c}-\mathrm{kit}^{+}$; MPP: CD150 CD $48^{-/ / 0}$ Lineage-Sca- $1^{+}$c-kit ${ }^{+} ; \quad$ HPC-1: CD150-CD $48^{+}$Lineage-Sca- ${ }^{+}$ c-kit ; HPC-2: CD $150^{+} \mathrm{CD} 48^{+}$Lineage-Sca- ${ }^{+} \mathrm{c}-\mathrm{kit}^{+}$; CMP: $\mathrm{CD} 34^{+}$

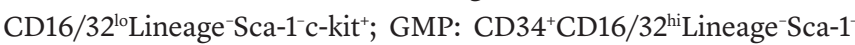
c-kit ${ }^{+}$; MEP: CD34 ${ }^{-/ \mathrm{lo}} \mathrm{CD} 16 / 32^{\mathrm{lo}}$ Lineage-Sca-1 ${ }^{-}{ }^{\mathrm{c}}-\mathrm{kit}^{+}$; Mac (monocyte/ macrophage): Mac-1+ $1^{+}-1^{-} \mathrm{B}^{2} 2 \mathrm{O}^{-} \mathrm{CD}^{-}$; Ery (erythroid progenitor): CD71 ${ }^{+}$Ter119 ${ }^{+} \mathrm{CD}_{4}{ }^{+}$; B cell: $\mathrm{B} 220^{+} \mathrm{CD}^{-}{ }^{-} \mathrm{Mac}-1^{-} \mathrm{Gr}-1^{-}$; T cell: $\mathrm{CD}^{+}$ B220-Mac-1- ${ }^{-} \mathrm{Gr}-1^{-}$; myeloid: Mac-1 ${ }^{+} \mathrm{B} 220^{-} \mathrm{CD} 3^{-}$; BMSC (bone marrow stromal cell): CD $45^{-}$Ter119- ${ }^{-} D 31^{-} \mathrm{CD} 140 \mathrm{a}^{+} \mathrm{CD} 105^{+}$.

Cell-cycle analysis. BrdU incorporation in vivo was measured by flow cytometry using the APC BrdU Flow Kit (BD Biosciences). Mice were given an intraperitoneal injection of $100 \mathrm{mg} / \mathrm{kg}$ BrdU (SigmaAldrich) in PBS and maintained on $1 \mathrm{mg} / \mathrm{ml} \mathrm{BrdU}$ in the drinking water for the indicated periods of time.

Competitive reconstitution assay. Adult recipient mice (CD45.1) were irradiated using an XRAD $320 \mathrm{X}$-ray irradiator (Precision $\mathrm{X}$-Ray) to deliver two equal doses of $5.4 \mathrm{~Gy}$ at least 3 hours apart. $10^{6}$ donor WBM cells from CD $45.2^{+}$Esr1 ${ }^{f / f l}$; Vav1-icre mice were transplanted along with $10^{6}$ competitor WBM cells from CD45.1 ${ }^{+}$WT mice into the tail vein of recipient mice. Sixteen weeks after transplantation, recipient mice were treated with $20 \mathrm{mg} / \mathrm{kg} / \mathrm{d} 27 \mathrm{HC}$ or vehicle for 6 days.

Colony formation assay. Peripheral blood mononuclear cells were isolated by Ficoll-Paque Premium (GE Healthcare) according to the manufacturer's instructions and were seeded in MethoCult GF M3434 methylcellulose culture medium (StemCell Technologies) supplemented with $10 \mathrm{ng} / \mathrm{ml}$ thrombopoietin (Peprotech). Colonies were counted after 10 days.

Quantitative reverse transcription PCR. HSCs and other hematopoietic cells were sorted into TRIzol LS Reagent (Thermo Fisher Scientific), and RNA was isolated using an RNeasy MinElute Cleanup Kit (QIAGEN) according to the manufacturer's instructions. cDNA was made with iScript Reverse Transcription Supermix for RT-qPCR (Bio-Rad Laboratories). Quantitative PCR was performed using iTaq Universal SYBR Green Supermix (Bio-Rad Laboratories) and a LightCycler 480 (Roche Life Science). Each sample was normalized to beta-Actin. Primers to quantify cDNA levels were as follows: Cyp27a1 forward: AGGGCAAGTACCCAATAAGAGA; Cyp27a1 reverse: TCGTTTAAGGCATCCGTGTAGA; Cxcr4 forward: ATGGAACCGATCAGTGTGAGT; Cxcr4 reverse: CCGACTATGCCAGTCAAGAAG; $\beta$-actin forward: GGCTGTATTCCCCTCCATCG; $\beta$-actin reverse: CCAGTTGGTAACAATGCCATGT.

Measurement of $27 \mathrm{HC}$ levels. Bone marrow lineage-depleted cells were obtained by incubating bone marrow cells with biotinylated antibodies against lineage markers, followed by anti-biotin microbeads, and Lineage ${ }^{+}$cells were depleted using an autoMACS Pro Separator (Miltenyi Biotec). 27HC was measured by LC-MS using a Sciex API 5000 triple quadrupole mass spectrometer (MS), as previously described (39).

Bleeding of mice. EMH was induced by bleeding mice 5 times over a 2-week period (every 3 days) (20). Approximately $300 \mu$ l blood was removed at each bleed. Mice were analyzed 2 days after the fifth bleed.

Statistics. Data represent mean \pm SD. Numbers of experiments noted in figure legends reflect independent experiments performed on different days. The sample size used in each experiment was not formally justified for statistical power. Blinding was not used. Mice were allocated to experiments randomly and samples processed in an arbitrary order, but formal randomization techniques were not used. For analysis of the statistical significance of differences between two groups, we performed 2-tailed unpaired Student's $t$ tests when groups were normally distributed and equally variable. Shapiro-Wilk normality tests and F-tests were used to check normality and variability, respectively. When data significantly violated normality and equal variances assumptions, they were $\log _{2}$ transformed and further tested for normality and variability. When transformed groups were normal but variances were unequal, Welch's tests were used. When transformed groups were not normal, Mann-Whitney $U$ tests were used. For analysis of the differences among more than two groups, when groups were normally distributed and equally variable, we performed 1-way ANOVA tests with Šídák's multiple comparisons tests taking each cell population as one family. Shapiro-Wilk normality tests and Levene's median tests were used to check normality and variability, respectively. When data significantly violated normality and equal variance assumptions, they were $\log _{2}$ transformed and further tested for normality and variability. When transformed groups still violated those assumptions, Kruskal-Wallis tests and Dunn's multiple comparisons tests were used as an alternative to 1-way ANOVA and Šídák's multiple comparisons tests. All statistical tests were performed using the GraphPad Prism software.

Study approval. All animal protocols were approved by the University of Texas Southwestern Medical Center Institutional Animal Care and Use Committee.

\section{Author contributions}

HO designed and performed most experiments. JGM quantified $27 \mathrm{HC}$ levels. ZZ performed statistical analyses. MU and PWS provided materials and advice on experiments. $\mathrm{HO}$ and SJM designed and interpreted the experiments and wrote the manuscript.

\section{Acknowledgments}

SJM is a Howard Hughes Medical Institute (HHMI) Investigator, the Mary McDermott Cook Chair in Pediatric Genetics, the Kathryne and Gene Bishop Distinguished Chair in Pediatric Research, director of the Hamon Laboratory for Stem Cells and Cancer, and a Cancer Prevention and Research Institute of Texas Scholar. HO was supported by a Japan Society for the Promotion of Science Postdoctoral Fellowship. PWS was supported by NIH HL087564. We thank Sohaib A. Khan for sharing Esr $1^{f / f l}$ mice; Bonne Thompson for measuring 27HC levels; Nicolas Loof and the Moody Foundation Flow Cytometry Facility; Kristen Correll, Albert Gross, and Ashley Leach for mouse colony management; and Michalis Agathocleous, Stefano Comazzetto, and Le Qi for technical assistance and advice.

Address correspondence to: Hideyuki Oguro, The Jackson Laboratory for Genomic Medicine, 10 Discovery Drive, Farmington, Connecticut 06032, USA. Phone: 860.837.2052; Email: Hideyuki.Oguro@jax.org. Or to: Sean J. Morrison, University of Texas Southwestern Medical Center, 5323 Harry Hines Boulevard, Dallas, Texas 75390, USA. Phone: 214.648.7515; Email: Sean.Morrison@UTSouthwestern.edu. 
1. Cottler-Fox MH, et al. Stem cell mobilization. Hematology Am Soc Hematol Educ Program. 2003;2003(1):419-437.

2. Nakada D, et al. Oestrogen increases haematopoietic stem-cell self-renewal in females and during pregnancy. Nature. 2014;505(7484):555-558.

3. Inra $\mathrm{CN}$, et al. A perisinusoidal niche for extramedullary haematopoiesis in the spleen. Nature. 2015;527(7579):466-471.

4. Umetani M, et al. 27-Hydroxycholesterol is an endogenous SERM that inhibits the cardiovascular effects of estrogen. Nat Med. 2007;13(10):1185-1192.

5. Russell DW. The enzymes, regulation, and genetics of bile acid synthesis. Annu Rev Biochem. 2003;72:137-174.

6. Karuna R, et al. Plasma levels of 27-hydroxycholesterol in humans and mice with monogenic disturbances of high density lipoprotein metabolism. Atherosclerosis. 2011;214(2):448-455.

7. Rosen $\mathrm{H}$, et al. Markedly reduced bile acid synthesis but maintained levels of cholesterol and vitamin $\mathrm{D}$ metabolites in mice with disrupted sterol 27-hydroxylase gene. J Biol Chem. 1998;273(24):14805-14812.

8. Wu Q, et al. 27-Hydroxycholesterol promotes cell-autonomous, ER-positive breast cancer growth. Cell Rep. 2013;5(3):637-645.

9. Nelson ER, et al. 27-Hydroxycholesterol links hypercholesterolemia and breast cancer pathophysiology. Science. 2013;342(6162):1094-1098.

10. Umetani $\mathrm{M}$, et al. The cholesterol metabolite 27-hydroxycholesterol promotes atherosclerosis via proinflammatory processes mediated by estrogen receptor alpha. Cell Metab. 2014;20(1):172-182.

11. Gomes AL, Carvalho T, Serpa J, Torre C, Dias S. Hypercholesterolemia promotes bone marrow cell mobilization by perturbing the SDF1:CXCR4 axis. Blood. 2010;115(19):3886-3894.

12. Murphy AJ, et al. ApoE regulates hematopoietic stem cell proliferation, monocytosis, and monocyte accumulation in atherosclerotic lesions in mice. JClin Invest. 2011;121(10):4138-4149.

13. Feng Y, et al. Hematopoietic stem/progenitor cell proliferation and differentiation is differentially regulated by high-density and low-density lipoproteins in mice. PLoS One. 2012;7(11):e47286.

14. Crysandt M, et al. Hypercholesterolemia and its association with enhanced stem cell mobilization and harvest after high-dose cyclophosphamide+G-CSF. Bone Marrow Trans- plant. 2011;46(11):1426-1429.

15. Westerterp M, et al. Regulation of hematopoietic stem and progenitor cell mobilization by cholesterol efflux pathways. Cell Stem Cell. 2012;11(2):195-206.

16. Yvan-Charvet L, et al. ATP-binding cassette transporters and HDL suppress hematopoietic stem cell proliferation. Science. 2010;328(5986):1689-1693.

17. Oguro H, Ding L, Morrison SJ. SLAM family markers resolve functionally distinct subpopulations of hematopoietic stem cells and multipotent progenitors. Cell Stem Cell. 2013;13(1):102-116.

18. Akashi K, Traver D, Miyamoto T, Weissman IL. A clonogenic common myeloid progenitor that gives rise to all myeloid lineages. Nature. 2000;404(6774):193-197.

19. Sánchez-Aguilera A, et al. Estrogen signaling selectively induces apoptosis of hematopoietic progenitors and myeloid neoplasms without harming steady-state hematopoiesis. Cell Stem Cell. 2014;15(6):791-804.

20. Cheshier SH, Prohaska SS, Weissman IL. The effect of bleeding on hematopoietic stem cell cycling and self-renewal. Stem Cells Dev. 2007;16(5):707-717.

21. Basaran A. Pregnancy-induced hyperlipoproteinemia: review of the literature. Reprod Sci. 2009;16(5):431-437.

22. Nie Y, Han YC, Zou YR. CXCR4 is required for the quiescence of primitive hematopoietic cells. JExp Med.2008;205(4):777-783.

23. Broxmeyer HE, et al. Rapid mobilization of murine and human hematopoietic stem and progenitor cells with AMD3100, a CXCR4 antagonist. J Exp Med. 2005;201(8):1307-1318.

24. Tzeng YS, Li H, Kang YL, Chen WC, Cheng WC, Lai DM. Loss of Cxcl12/Sdf-1 in adult mice decreases the quiescent state of hematopoietic stem/progenitor cells and alters the pattern of hematopoietic regeneration after myelosuppression. Blood. 2011;117(2):429-439.

25. Gamba L, Cubedo N, Ghysen A, Lutfalla G, Dambly-Chaudière C. Estrogen receptor ESR1 controls cell migration by repressing chemokine receptor CXCR4 in the zebrafish posterior lateral line system. Proc Natl Acad Sci U S A. 2010;107(14):6358-6363.

26. Schwartz JA, Skafar DF. Ligand-mediated modulation of estrogen receptor conformation by estradiol analogs. Biochemistry. 1993;32(38):10109-10115.

27. Paige LA, et al. Estrogen receptor (ER) modula- tors each induce distinct conformational changes in ER alpha and ER beta. Proc Natl Acad Sci U S A. 1999;96(7):3999-4004.

28. Bruning JB, et al. Coupling of receptor conformation and ligand orientation determine graded activity. Nat Chem Biol. 2010;6(11):837-843.

29. Wardell SE, Kazmin D, McDonnell DP. Research resource: transcriptional profiling in a cellular model of breast cancer reveals functional and mechanistic differences between clinically relevant SERM and between SERM/estrogen complexes. Mol Endocrinol. 2012;26(7):1235-1248.

30. Caizzi L, et al. Genome-wide activity of unliganded estrogen receptor- $\alpha$ in breast cancer cells. Proc Natl Acad Sci U S A. 2014;111(13):4892-4897.

31. DuSell CD, Umetani M, Shaul PW, Mangelsdorf DJ, McDonnell DP. 27-hydroxycholesterol is an endogenous selective estrogen receptor modulator. Mol Endocrinol. 2008;22(1):65-77.

32. Feng Q, O'Malley BW. Nuclear receptor modulation - role of coregulators in selective estrogen receptor modulator (SERM) actions. Steroids. 2014;90:39-43

33. Sigurdsson V, et al. Bile acids protect expanding hematopoietic stem cells from unfolded protein stress in fetal liver. Cell Stem Cell. 2016;18(4):522-532.

34. Cimato TR, Palka BA, Lang JK, Young RF. LDL cholesterol modulates human CD34+ HSPCs through effects on proliferation and the IL-17 G-CSF axis. PLoS One. 2013;8(8):e73861.

35. Ordovas JM, Pocovi M, Grande F. Plasma lipids and cholesterol esterification rate during pregnancy. Obstet Gynecol. 1984;63(1):20-25

36. Feng Y, Manka D, Wagner KU, Khan SA. Estrogen receptor-alpha expression in the mammary epithelium is required for ductal and alveolar morphogenesis in mice. Proc Natl Acad Sci U S A. 2007;104(37):14718-14723.

37. de Boer J, et al. Transgenic mice with hematopoietic and lymphoid specific expression of Cre. Eur JImmunol. 2003;33(2):314-325.

38. Lieschke GJ, et al. Mice lacking granulocyte colony-stimulating factor have chronic neutropenia, granulocyte and macrophage progenitor cell deficiency, and impaired neutrophil mobilization. Blood.1994;84(6):1737-1746

39. McDonald JG, Smith DD, Stiles AR, Russell DW. A comprehensive method for extraction and quantitative analysis of sterols and secosteroids from human plasma. J Lipid Res. 2012;53(7):1399-1409. 